\title{
Removal of a Cationic Dye by Adsorption/Photodegradation Using Electrospun PAN/O-MMT Composite Nanofibrous Membranes Coated with $\mathrm{TiO}_{2}$
}

\author{
Qingqing Wang, ${ }^{1}$ Dawei Gao, ${ }^{1}$ Chuntao Gao, ${ }^{1}$ Qufu Wei, ${ }^{1}$ \\ Yibing Cai, ${ }^{1}$ Jing $\mathrm{Xu},{ }^{2}$ Xiaoya Liu, ${ }^{2}$ and Yang $\mathrm{Xu}^{1}$ \\ ${ }^{1}$ Key Laboratory of Eco-Textiles, Ministry of Education, Jiangnan University, Wuxi 214122, China \\ ${ }^{2}$ School of Chemical and Material Engineering, Jiangnan University, Wuxi 214122, China \\ Correspondence should be addressed to Qufu Wei, qufu_wei@163.com
}

Received 8 September 2011; Revised 5 November 2011; Accepted 14 November 2011

Academic Editor: Mietek Jaroniec

Copyright ( 2012 Qingqing Wang et al. This is an open access article distributed under the Creative Commons Attribution License, which permits unrestricted use, distribution, and reproduction in any medium, provided the original work is properly cited.

\begin{abstract}
Polyacrylonitrile (PAN)/organic-modified montmorillonite (O-MMT) composite nanofibrous membranes were firstly prepared by electrospinning and then coated with titanium dioxide $\left(\mathrm{TiO}_{2}\right)$ using spin coating technique. The structural morphology of the nanofibrous membranes with different mass ratio of O-MMT before and after spin coating was investigated by scanning electron microscope (SEM) and transmission electron microscope (TEM). The chemical property of adsorbed methylene blue (MB) was analyzed by infrared spectroscopy (IR). The adsorption and photodegradation capability of the $\mathrm{TiO}_{2}$-coated PAN/OMMT composite nanofibrous membranes were evaluated by adsorption rate of MB and K/S values of the membranes before and after UV irradiation. The experimental results indicated that with the increase of O-MMT amount, the diameters of the nanofibers decreased and the adsorption rate of $\mathrm{MB}$ was evidently improved. Besides, with the increase of $\mathrm{TiO}_{2}$ film layers, the photocatalytic properties were enhanced while the adsorption process was slowed down.
\end{abstract}

\section{Introduction}

Industrial dyes have been recognized as one of the largest sources of water contamination. Some of the dyes and their metabolites are toxic and carcinogenic and reported to have connections with different respiratory disorders worldwide [1]. Many efforts have been devoted in recent years to remove dyes from wastewaters, such as carbon adsorption, filtration, chemical precipitation, photodegradation, biodegradation, electrolytic chemical treatment, and membrane technology [2]. Adsorption removal is an effective and simple method for dye treatment but usually produces large amount of sludge [3], which may cause secondary pollution. As is known, no individual treatment technique is ideal enough to efficiently remove dyes from wastewaters with no secondary contaminants generated. So combining two or more treatment techniques together seems quite necessary.

So far, activated carbon is the most widely used adsorbent for organic substances, including dyes, due to its high adsorption capacity, high specific surface area, and low selectivity [4]. However, it is too expensive. Hence, lots of studies have investigated alternative adsorbents with high efficiency for dye removal at a reasonable cost [5-7]. Electrospun nanofibrous membrane is a good choice for it possesses a number of interesting characteristics such as high porosity, large surface area per unit mass, high gas permeability, and small interfibrous porous size [8]. The introduction of specific functional groups onto the electrospun nanofibers $[9,10]$ or addition of inorganic nanoparticles like montmorillonite (MMT) [11] into polymer matrix was reported for the removal or adsorption of specific materials from aqueous solutions. Some recent studies $[12,13]$ indicated that MMT clay has been accepted as one of the low-cost adsorbents for cationic dye adsorption because there are plenty of negative charges on MMT clays and it has large cation-exchange capacity (CEC). However, to the best of our knowledge, there is no literature focusing on the adsorption capacity of cationic dye onto the electrospun nanofibers based on 
polymers and MMT clays. PAN is easily to be electrospun into nanofibers and it is stable in the environment. So in this work, we studied the adsorption properties of methylene blue (MB) onto the electrospun PAN/MMT composite nanofibers.

As we have mentioned herein before, adsorption process would result in secondary pollution, so $\mathrm{TiO}_{2}$ was introduced onto the composite nanofibrous membrane by spin coating. Due to its chemical stability, nontoxicity, and high photocatalytic reactivity, $\mathrm{TiO}_{2}$ has been recognized as the best candidate for photocatalytic applications $[14,15]$. A low temperature process is adopted because it is beneficial for the use of low thermally resistant materials such as plastics, wood, or paper as substrates [16].

In this paper, low-temperature $\mathrm{TiO}_{2}$ was synthesized and spin coated onto the surface of electrospun PAN/OMMT composite nanofibrous membrane. The adsorption and photocatalysis processes were investigated, and the effects of O-MMT concentration and spin coating layers on adsorption capacity and photocatalytic efficiency of the composite membranes were evaluated through the adsorption and degradation of $\mathrm{MB}$ from aqueous solution.

\section{Experimental}

2.1. Materials. The montmorillonite (MMT, cation exchange capacity, $97 \mathrm{meq} / 100 \mathrm{~g}$ of clay) organically modified by cetyltrimethyl ammonium bromide (CTAB) was a kind gift from Zhejiang Fenghong Clay Chemicals Co., Ltd. The average thickness of the O-MMT was less than $25 \mathrm{~nm}$, and the ratio of diameter to thickness was about 200. The polyacrylonitrile (PAN, Mw $=79,100$ ) powder was obtained from Aldrich. The 99.5\% N, N-dimethyl formamide (DMF), tetrabutyl titanate $\left(\mathrm{Ti}\left(\mathrm{OC}_{4} \mathrm{H}_{9}\right)_{4}, \mathrm{CP}\right)$, ethanol $(\mathrm{EtOH}, \mathrm{AR})$ and hydrochloric acid $(\mathrm{HCl}, \mathrm{AR})$ were all used as received.

2.2. Preparation of Electrospun Composite Nanofibers. $3 \mathrm{~g}$ PAN powder was dissolved in $27 \mathrm{~mL}$ DMF by magnetically stirring for $24 \mathrm{hrs}$ at room temperature. Then the polymer solution was centrifuged at the speed of $4000 \mathrm{rpm}$ for $10 \mathrm{~min}$ to remove the impurities. After the purification process, certain quantity of O-MMT powders was added into the polymer solution and stirred another $6 \mathrm{~h}$ to prepare electrospinning solutions with $0,1,3,5 \mathrm{wt}$. $\%$ O-MMT, respectively.

The polymer solutions were electrospun at a positive voltage of $12 \mathrm{kv}$ with a working distance of $15 \mathrm{~cm}$, and the flowrate was set as $0.5 \mathrm{~mL} / \mathrm{h}$.

2.3. Synthesis of Low-Temperature $\mathrm{TiO}_{2}$. The low-temperature $\mathrm{TiO}_{2}$ sol was prepared by firstly dissolving $10 \mathrm{~mL}$ $\mathrm{Ti}\left(\mathrm{OC}_{4} \mathrm{H}_{9}\right)_{4}$ in $5 \mathrm{~mL}$ absolute ethanol. After magnetically stirring for a certain time at room temperature until it was completely homogeneous, the solution was added dropwise into the blended solution of deionized water and HCl. The mixtures were placed at room temperature for 5 days to obtain transparent $\mathrm{TiO}_{2}$ sol.

2.4. Spin Coating Technique. The PAN and PAN/O-MMT composite nanofibrous membranes were cut into $7 \mathrm{~cm} * 7 \mathrm{~cm}$

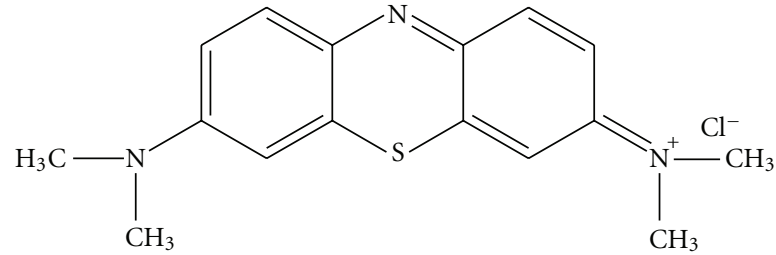

FIgUre 1: The chemical structure of methylene blue.

squares. The aluminum foil side was pasted on the disc. An amount of $0.1 \mathrm{~mL} \mathrm{TiO}$ sol was spin coated on the surface of the nanofibrous membranes at the speed of $4000 \mathrm{rpm}$. The membranes coated with $\mathrm{TiO}_{2}$ were dried at $60^{\circ} \mathrm{C}$ in vacuum oven for $10 \mathrm{~min}$.

2.5. Adsorption and Photocatalytic Degradation of $M B$. The adsorption performance of the PAN and PAN/O-MMT composite nanofiber membranes coated with $\mathrm{TiO}_{2}$ was evaluated through the decreased UV absorbency of $\mathrm{MB}$ solution. The chemical structure of $\mathrm{MB}$ is shown in Figure 1. The concentration of MB was $0.02 \mathrm{~g} / \mathrm{L}$. The samples weighed $0.05 \mathrm{~g}$ were placed in beakers containing $40 \mathrm{~mL} \mathrm{MB}$ solution, and the absorbency of the MB solution at the wavelength of $664 \mathrm{~nm}$ was measured every $10 \mathrm{~min}$ by a UV-vis spectrophotometer (UV-2100, China). The adsorption rate of MB can be calculated using the following formula:

$$
\text { Adsorption rate }=\frac{A_{0}-A}{A_{0}},
$$

where $A_{0}$ and $A$ are the absorbency of the initial and remaining $\mathrm{MB}$, respectively.

The photocatalytic performance of the membranes was evaluated by an Xrite- 8400 spectrophotometer under illuminant D65 using the $10^{\circ}$ standard observer [17]. The relative color strength and staining on the white nanofibrous membrane were determined with the Kubelka-Munk equations. The color strength was signified by the K/S value,

$$
\frac{K}{S}=\frac{1-R^{2}}{2 R},
$$

where $K$ is the absorption coefficient, $S$ is the scattering coefficient, and $R$ is the fraction reflectance (value from 0 to 1 ) of the dyed substrate at the wavelength of minimum reflectance. The $K / S$ values are proportional to the dyeing color [18].

After adsorption process, the nanofibrous membranes were dried and the $K / S$ value was measured. Then the membranes were placed in beakers with $40 \mathrm{~mL}$ deionized water under $100 \mathrm{w}$ ultraviolet (UV) light at a wavelength of $254 \mathrm{~nm}$. After 48 hour's irradiation, the membranes were taken out and dried for measuring the $K / S$ value.

2.6. Characterization. Scanning electron microscope (SEM, Quanta 200, Holland FEI Company) was used to investigate the surface morphology of the PAN and PAN/O-MMT composite nanofibers before and after spin coating. The samples were coated with a thin layer of gold by sputtering before the SEM imaging. Diameters of the electrospun nanofibers 

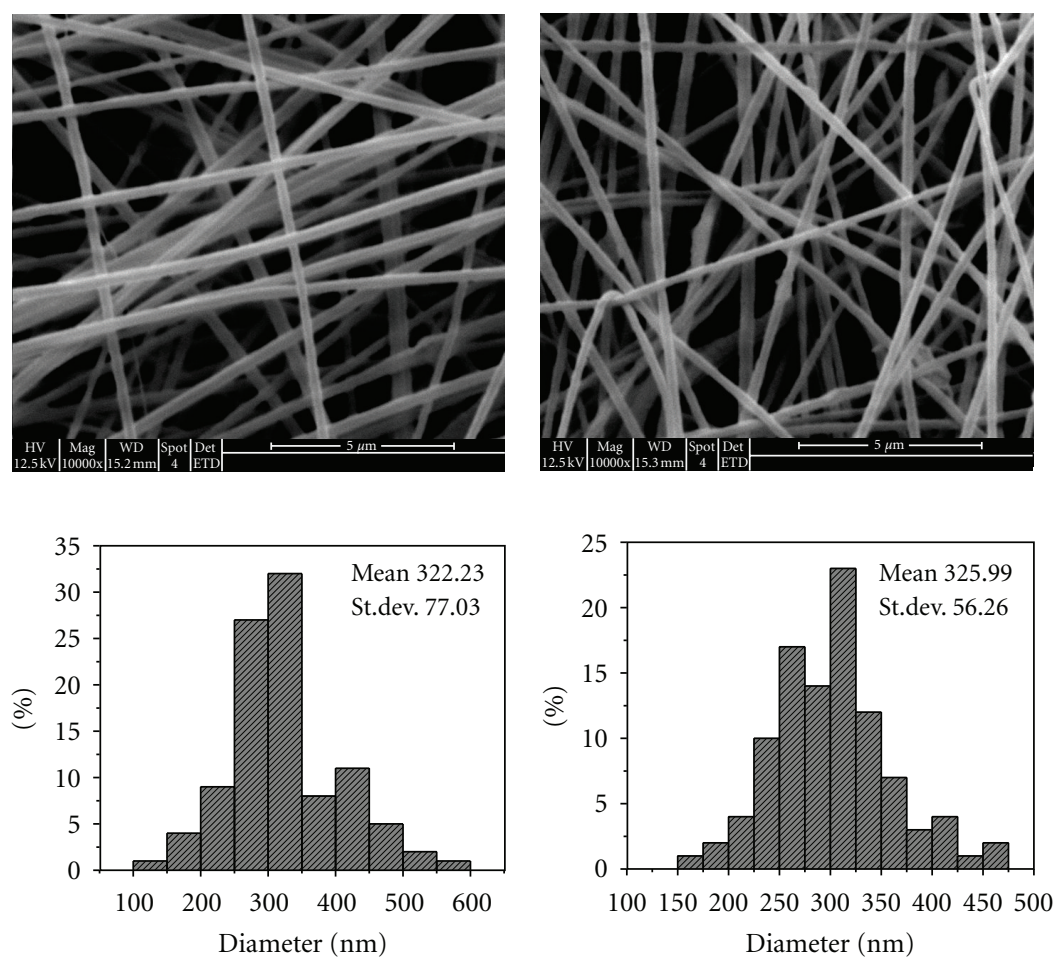

(a)

(b)
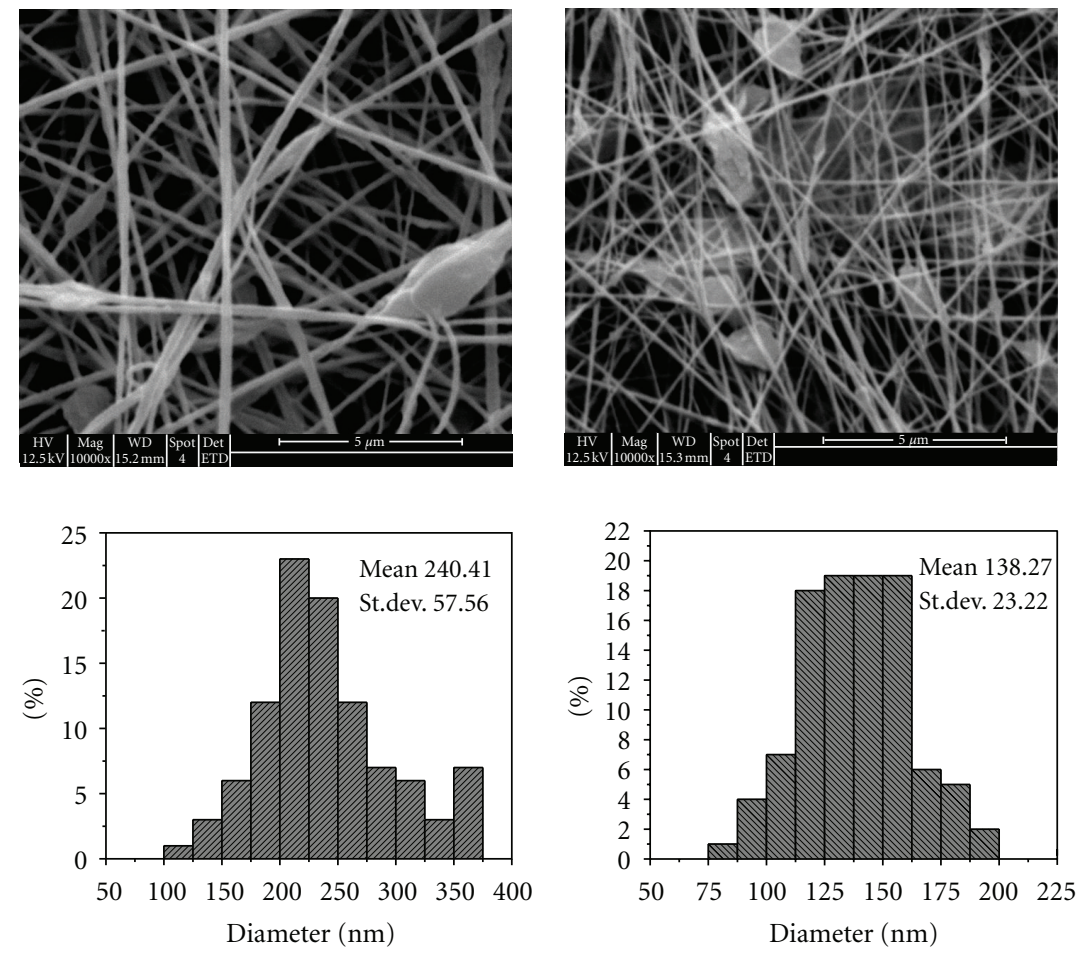

(c)

(d)

FIGURE 2: SEM images and diameter histogram distribution chart of PAN and PAN/O-MMT composite nanofibers: (a) 0 wt.\% O-MMT; (b) 1 wt.\% O-MMT; (c) 3 wt.\% O-MMT; (d) 5 wt.\% O-MMT.

were measured by Adobe Acrobat 7.0 professional from the SEM images, and 100 fibers were analyzed for each sample to obtain an average fiber diameter. In detail, after the SEM images were opened by Adobe Acrobat professional, the distance measurement was clicked to measure the diameter of the nanofibers. The zoom factor was set according to the scale plate in the SEM images before measuring. Every time the diameters were measured, a serial number was given to 


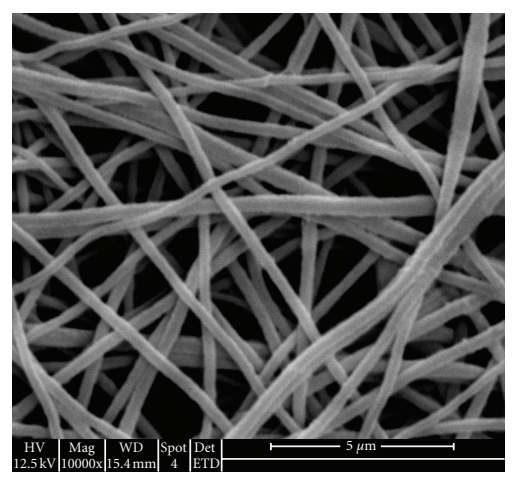

(a)

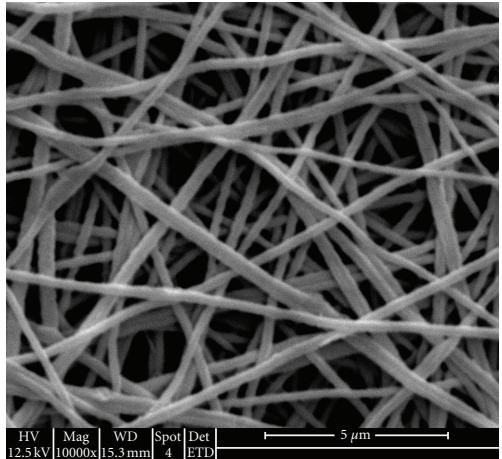

(b)

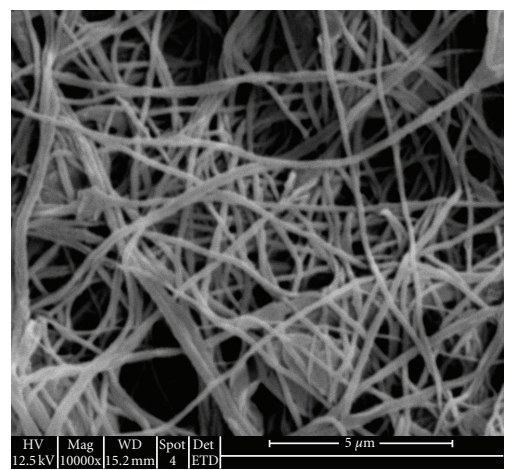

(c)

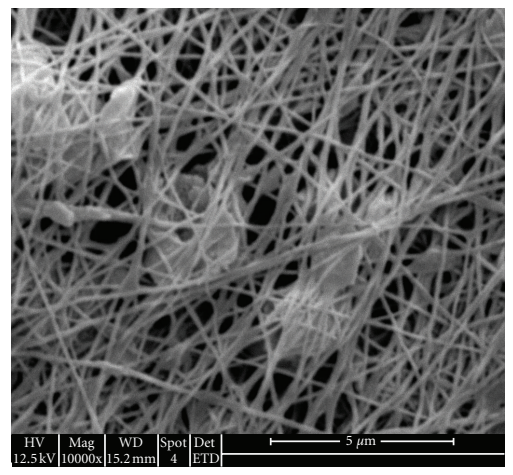

(d)

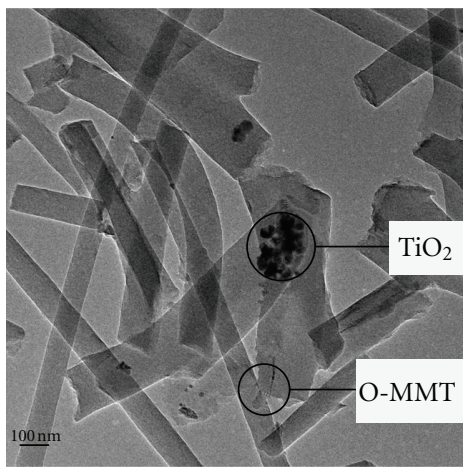

(e)

Figure 3: SEM (a-d) and TEM (e) images of PAN and PAN/O-MMT spin coated with $\mathrm{TiO}_{2}$ : (a) 0 wt.\% O-MMT; (b) 1 wt.\% O-MMT; (c) 3 wt.\% O-MMT; (d) 5 wt.\% O-MMT; (e) TEM of c.

the data obtained. Finally, the data were exported to origin professional 7.0 to analyze the diameter distribution.

Transmission electron microscope (TEM, JEOL2010, Philips) was employed to analyze the intercalation structure of the PAN/O-MMT composite nanofibers and the existence of $\mathrm{TiO}_{2}$ particles. The nanofibrous membranes after spin coating were pestled into powders and then dissolved in blend solution of deionized water and ethanol, using ultrasonic vibration to fully disperse the nanofibrous powders, and then dripped onto a 200-mesh Cu grid.

The intercalation structures of the MB in O-MMT layers after adsorption process were investigated by Fourier Transform Infrared Spectroscopy (FTIR, Nicolet Nexus, Thermo Electron Corporation) in the range $4000-400 \mathrm{~cm}^{-1}$, using $\mathrm{KBr}$-pressed method. The spectra were recorded with 32 scans with a resolution of $4 \mathrm{~cm}^{-1}$.

\section{Results and Discussion}

3.1. Structural Morphology of the As-Spun PAN and PAN/OMMT Composite Nanofibers. Morphologies of the electrospun nanofibers with different content of O-MMT were investigated by SEM, as shown in Figure 2. As can be seen from this figure, the morphology and average diameter of the electrospun PAN/O-MMT composite nanofibers are significantly affected by the amount of O-MMT added. With the increase of O-MMT loading, the average diameters of the composite nanofibers are decreased and some beaded structures are formed, as indicated in Figures 2(c) and 2(d). For pure PAN nanofibers, grooves can be seen on the surface of the most nanofibers, which might be caused by the solvent volatilization. The diameters of pure PAN nanofibers were ranged from 250 to $550 \mathrm{~nm}$, mostly distributed in $350-375 \mathrm{~nm}$, while the average diameter of the electrospun PAN composite nanofibers with $1 \mathrm{wt} . \%$ O-MMT was approximately $325 \mathrm{~nm}$, just slightly decreased compared to pure PAN nanofibers, and no sign of beaded formation, as indicated in Figure 2(b). When the amount of O-MMT increased to $3 \mathrm{wt} . \%$ and $5 \mathrm{wt} . \%$, the average diameters of the composite nanofibers were further decreased to $240 \mathrm{~nm}$ and $138 \mathrm{~nm}$, respectively. The beaded structures of the nanofibers were also formed, which could be attributed to the aggregation (multilayer stacks) of the O-MMT [19], as shown in Figures 2(c) and 2(d). The loading O-MMT containing a quaternary ammonium ion as an organic modifier and $\mathrm{Na}^{+}$and $\mathrm{Zn}^{2+}$ ions located between MMT layers improved the conductivity of PAN/O-MMT solutions. During the electrospinning process, the charged density in ejected jets was increased, and thus stronger elongation forces were imposed to the jets because of the self-repulsion of the excess charges under the electrical field, resulting in smaller diameters of the electrospun nanofibers [20].

\subsection{Surface Morphology of the Electrospun Nanofibers Coated} with $\mathrm{TiO}_{2}$. The surface morphology after spin coating was investigated by SEM, and TEM was employed to investigate the dispersion of O-MMT and $\mathrm{TiO}_{2}$. 


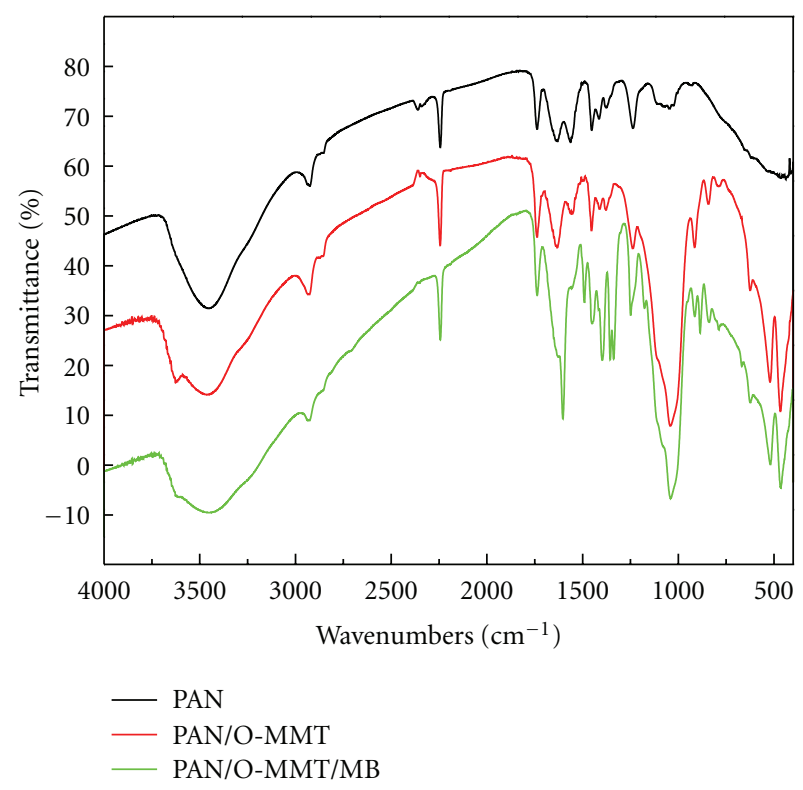

Figure 4: FTIR spectra of PAN, PAN/O-MMT nanofibers, and PAN/O-MMT nanofibers adsorbed with MB.

After spin coating, all fibers kept their fibrous shape, but became more densely packed and relaxed, as shown in Figures 3(a)-3(d). The nanofibers with smaller diameters tend to stick together. And so the pores generated by the nanofibers interwining together became smaller. This is in accordance with the experimental phenomenon. After spin coating, the membranes shrank and became stiffer.

Figure 3(e) shows the TEM micrograph of the PAN/OMMT composite nanofibers coated with $\mathrm{TiO}_{2}$ sol, in which the brighter fibers represent the PAN matrix and the dark narrow stripes as well as the particles stand for the O-MMT and $\mathrm{TiO}_{2}$, respectively. The O-MMT lamellas exfoliated and oriented along the fibers axis direction. This alignment might be attributed to the traction force generated by the electric field. Besides, the coated $\mathrm{TiO}_{2}$ sol clustered on the surface of the nanofibers but not uniformly distributed.

3.3. FTIR Analysis. The FTIR spectra of PAN, PAN/O-MMT nanofibers, and PAN/O-MMT nanofibers adsorbed with $\mathrm{MB}$ are presented in Figure 4. It can be seen from the PAN curve that the peak at $2242 \mathrm{~cm}^{-1}$ can be assigned to nitrile groups, while those at 2940 and $1452 \mathrm{~cm}^{-1}$ are, respectively, ascribed to the $\mathrm{C}-\mathrm{H}$ stretching vibration and bending vibration. Compared with the spectra of PAN nanofibers, PAN/O-MMT showed the development of additional bands at $3618,1037,838,518$, and $464 \mathrm{~cm}^{-1}$, corresponding to $\mathrm{Al}-\mathrm{O}$ stretching vibration, $\mathrm{Si}-\mathrm{O}$ stretching vibration, $\mathrm{Si}-\mathrm{O}-$ $\mathrm{H}$ stretching vibration, $\mathrm{Si}-\mathrm{O}-\mathrm{Al}$ stretching vibration, and Si-O-Si bending vibration of MMT, respectively. After MB adsorption process, some additional bands can also be seen from Figure 4. The bands at 2805 and $2709 \mathrm{~cm}^{-1}$ are assigned to symmetric and asymmetric stretching vibrations of methyl and methylene groups, whereas the characteristic peaks at 1604 and $1335 \mathrm{~cm}^{-1}$ are stretching vibrations of $\mathrm{C}=\mathrm{C}$ in aromatic rings and $\mathrm{C}-\mathrm{N}$ stretching vibrations of tertiary

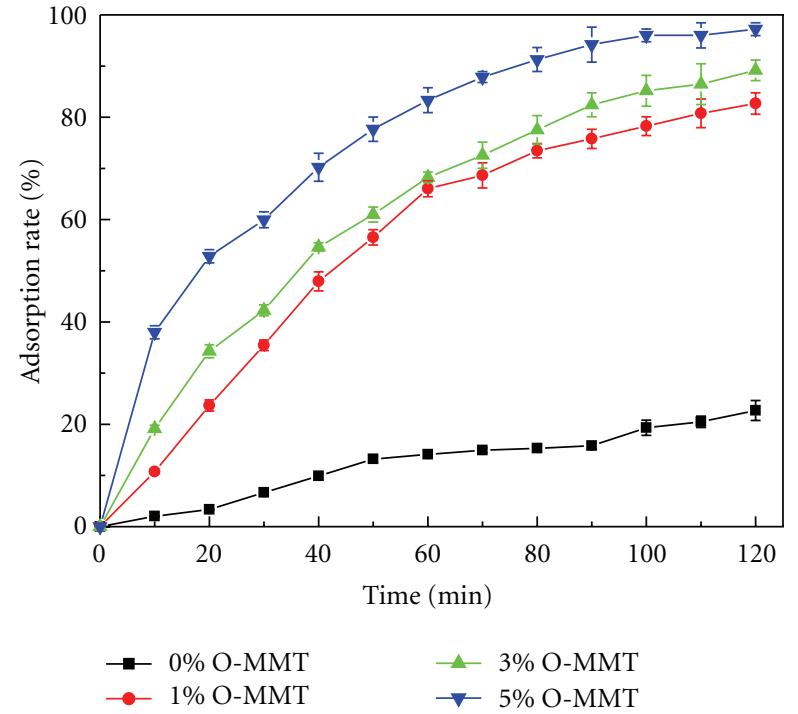

FIGURE 5: Effect of the amounts of O-MMT on adsorption capacity of the membranes for MB.

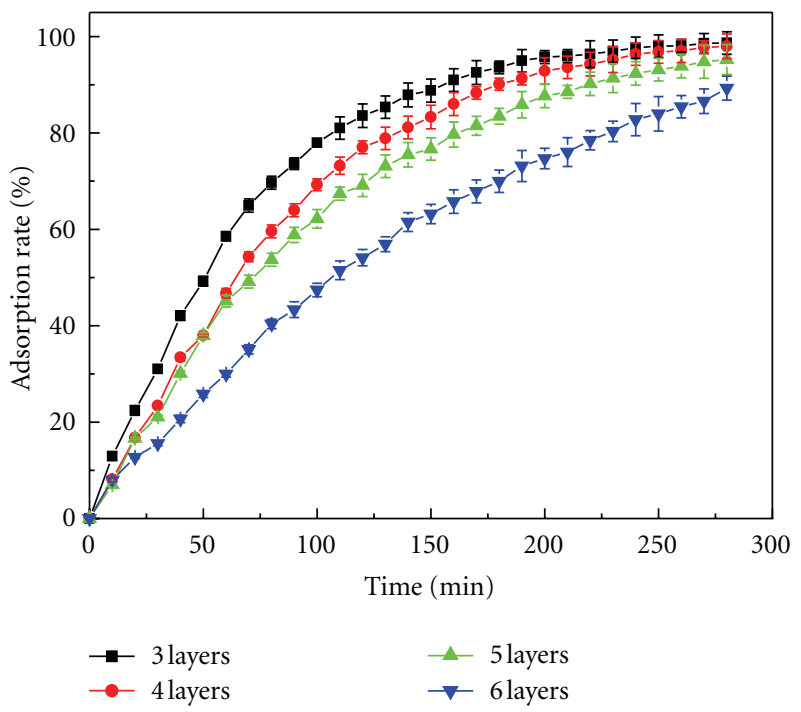

Figure 6: Effect of the $\mathrm{TiO}_{2}$ film layers on adsorption capacity of the membranes for MB: the substrate is PAN composite nanofibrous membranes with 3 wt.\% O-MMT.

amines of MB, respectively. A new IR band at $912 \mathrm{~cm}^{-1}$ is shown, which might be attributed to $\mathrm{Si}-\mathrm{O}^{\delta-\cdots \delta+}-\mathrm{MB}^{+}$from the polarized $\mathrm{Si}-\mathrm{O}$ bond, that is, $\mathrm{MB}$ combined with $\mathrm{SiO}_{2}$ in MMT. This confirms the intercalation of MB onto the MMT component of PAN/O-MMT composite nanofibers.

3.4. Adsorption Properties of the Membranes. The effects of different concentrations of O-MMT on adsorption capacity of PAN and PAN/O-MMT composite nanofibers for MB are shown in Figure 5. The error bars represent the standard deviation of three separate replicates of each experiment. It is clear that O-MMT content is an important factor affecting adsorption capacity of the membranes. As can be seen from Figure 5, the adsorption rates at $120 \mathrm{~min}$ of the four samples 


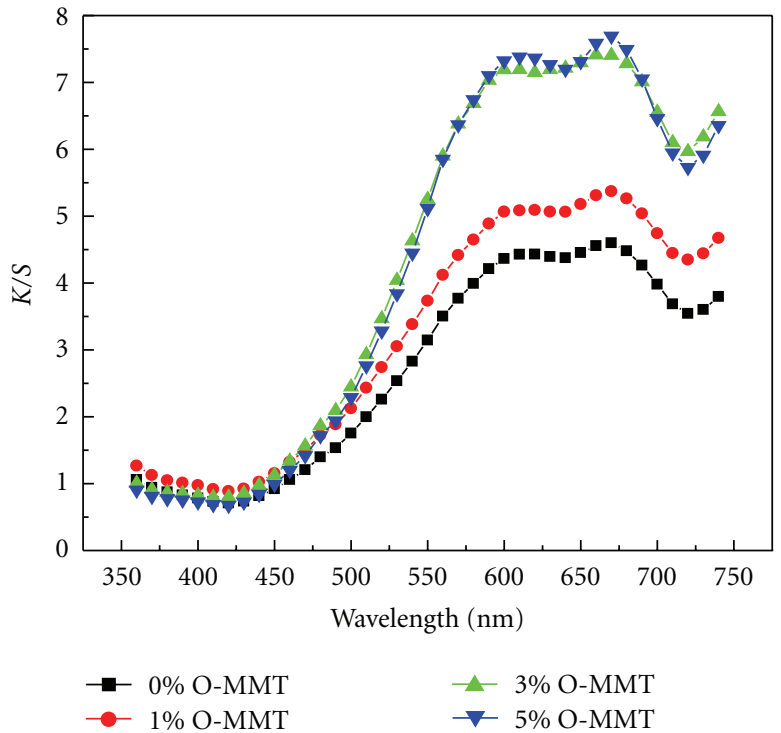

(a)

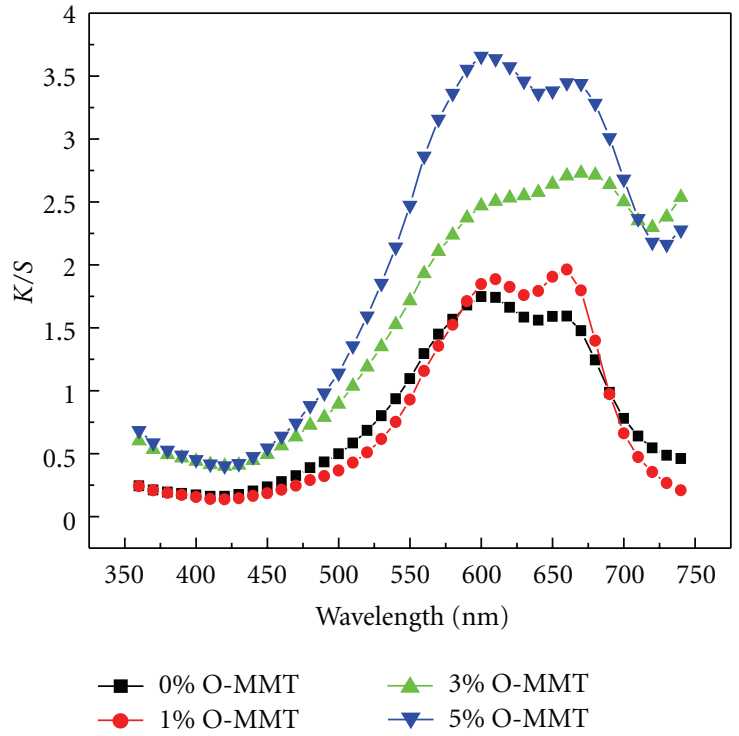

(b)

FIGURE 7: K/S curves of the PAN composite nanofibers with different mass ratios of O-MMT before (a) and after (b) photocatalytic process: the substrate is PAN/O-MMT composite nanofibrous membranes coated with three layers of $\mathrm{TiO}_{2}$.

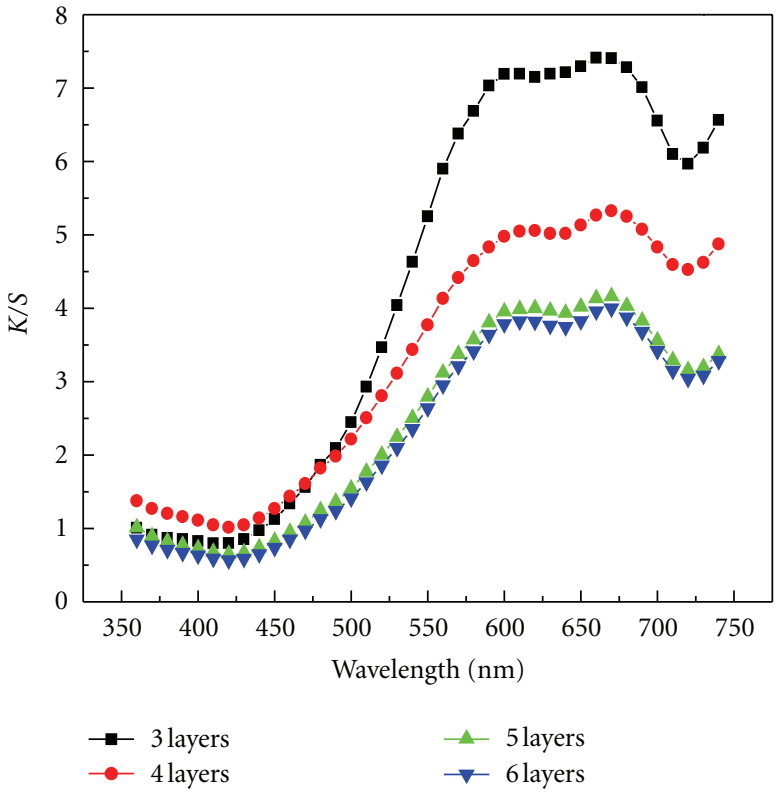

(a)

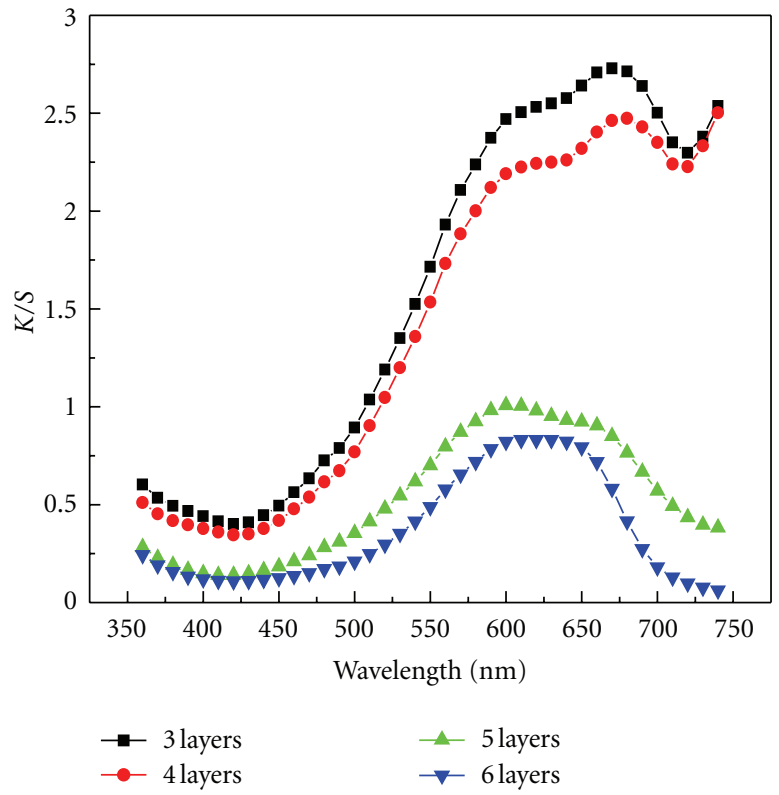

(b)

FIGURE 8: K/S curves of the membranes coated with different layers before (a) and after (b) photocatalytic process: the substrate is PAN composite nanofibrous membranes with 3\%O-MMT.

with $0,1,3$. and 5 wt.\% O-MMT were $22.69 \%, 82.69 \%$, $89.18 \%$, and $97.21 \%$, respectively. According to the SEM analysis, the addition of O-MMT decreased the nanofibers diameter, so the specific surface area was increased, resulting in higher MB adsorption rate. Besides, the introduced MMT generated a loose and porous surface [21]. This surface is convenient for the penetration of dye molecules into the composite nanofibers' surface and then may be of benefit to adsorption capacities of the membranes [22].
The effect of $\mathrm{TiO}_{2}$ film layers on adsorption capacities of PAN composite nanofibers with $3 \mathrm{wt}$ \% O-MMT for MB was also investigated, the results are shown in Figure 6, and the error bars represent the standard deviation of three separate replicates of each experiment. At the end of this experiment, the adsorption rates for the four samples coated with $3,4,5$, and 6 layers of $\mathrm{TiO}_{2}$ film were $98.66 \%, 97.98 \%$, $95.23 \%$, and $89.30 \%$, respectively. So the $\mathrm{TiO}_{2}$ coating layers have no significant effect on the adsorption capacities, but 

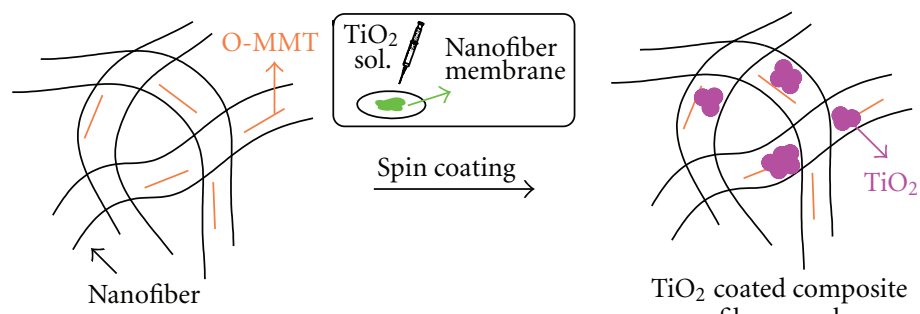

$\mathrm{TiO}_{2}$ coated composite nanofiber membrane
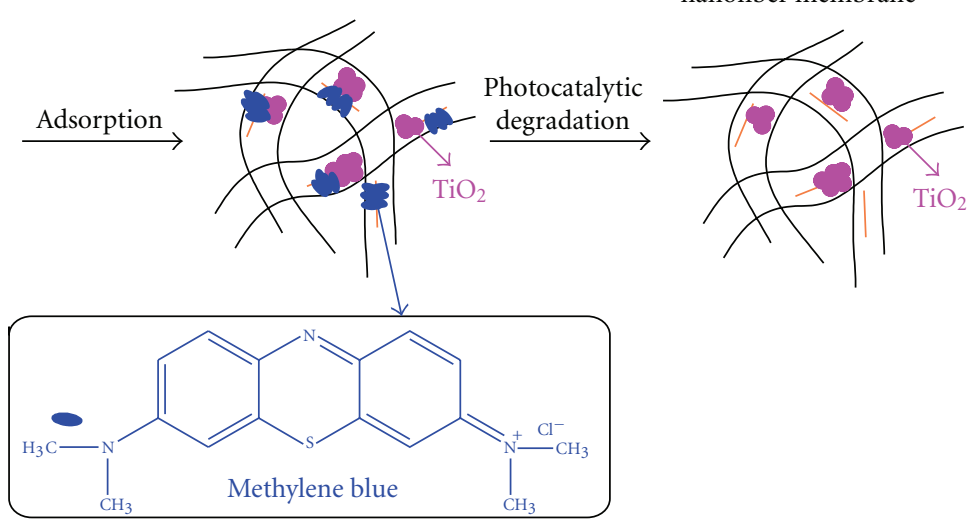

FIGURE 9: The flow chart of the formation of the composite membranes and the process of adsorption and photocatalytic degradation.

the adsorption process was slowed down. According to the SEM analysis, the nanofibers stick together and the pores among the nanofibers were narrowed or blocked after the spin coating process. Hence, the coating layers might hinder $\mathrm{MB}$ from contacting with O-MMT; thus it takes longer time to adsorb the same amount of $\mathrm{MB}$ than the uncoated membranes.

3.5. Photocatalytic Activity of the Composite Membranes. After adsorption process, the nanofiber membranes became blue with different degree of dye depth. K/S value is usually used to evaluate the depth of dying, so it was employed here to measure the degree of $\mathrm{MB}$ adsorption and photocatalytic degradation.

Before photocatalytic process, the membranes coated with three layers of $\mathrm{TiO}_{2}$ were immersed in $\mathrm{MB}$ solutions for $36 \mathrm{hrs}$ to reach adsorption equilibrium. Then the membranes were taken out and dried for K/S measurement; the corresponding K/S curves were shown in Figure 7(a). It can be seen that the K/S values for PAN composite nanofibers with $0,1,3$ and 5 wt.\% O-MMT were 4.5995, 5.3702, 7.4117, and 7.6924, respectively. The results indicated that the addition of O-MMT increased markedly the dye depth of nanofiber membranes.

After 48 hrs UV irradiation, the membranes became light blue. And the K/S values of the four samples decreased to $1.5913,1.9621,2.7069$, and 3.4409, respectively, as shown in Figure 7(b). It could be inferred that the O-MMT concentration only has effect on the adsorption capacity of O-MMT; however, the degradation rate of $\mathrm{MB}$ was not affected by the O-MMT content. Therefore, it can be concluded that the effect of O-MMT content on the photocatalytic degradation of $\mathrm{MB}$ adsorbed by the composite membranes could be neglected.
The number of $\mathrm{TiO}_{2}$ film layers has impact on the $\mathrm{MB}$ adsorption rate, as explained hereinbefore. However, the film layers may change the photocatalytic capacities evidently. So the effect of film layers on $\mathrm{MB}$ photodegradation is also investigated, as shown in Figure 8.

After adsorption process, the K/S values for the four membranes spin coated with 3,4,5, and 6 layers of $\mathrm{TiO}_{2}$ films were 7.4117, 5.3248, 4.1635, and 4.0003, respectively. As shown in Figure 8(a), membranes coated with 3 layers $\mathrm{TiO}_{2}$ films exhibited higher dye uptake rate. This phenomenon may be caused by $\mathrm{TiO}_{2}$ films' hindrance for the $\mathrm{MB}$ molecules to reach the ionic sites in MMT sheets.

Then, after $48 \mathrm{hrs}$ UV irradiation, the K/S values for the four samples became 2.7069, 2.4031, 0.9042, and 0.7183, respectively. The photocatalytic activity of the film was improved with the increased number of layers. When the $\mathrm{TiO}_{2}$ film grew thicker, the film contained more $\mathrm{TiO}_{2}$ catalyst. Therefore, the $\mathrm{TiO}_{2}$ particles continuously hydrolyzed and produced more oxidative groups such as $\cdot \mathrm{OH}, \cdot \mathrm{O}^{2-}$, and $\cdot \mathrm{OOH}$, and so catalytic activity of $\mathrm{TiO}_{2}$ thin film was accelerated.

3.6. Mechanisms of Adsorption and Photocatalytic Degradation. The preparation of the composite membrane spin coated by $\mathrm{TiO}_{2}$ and its use in $\mathrm{MB}$ adsorption and degradation are illustrated in Figure 9. As is seen, the as-spun PAN/OMMT nanofibers with intercalated structure were fabricated by electrospinning, and the O-MMT was oriented along the fiber axis direction. After spin coating, the $\mathrm{TiO}_{2}$ particles were randomly distributed on the surface of nanofibers. Then during the process of adsorption, part of the $\mathrm{MB}$ molecules was entrapped in the pores of the nanofibrous network, and some of the MB molecules were intercalated into interlayers of the MMT sheets, lying parallel to 
the aluminosilicate layers [23]. When the membranes were exposed to UV irradiation, the $\mathrm{TiO}_{2}$ particles were continuously hydrolyzed and produced large quantities of oxidative groups such as $\cdot \mathrm{OH}, \cdot \mathrm{O}^{2-}$, and $\cdot \mathrm{OOH}$, which accelerated the photodegradation of MB molecules. And so the membranes could be recycled and no secondary pollution was generated.

\section{Conclusion}

The PAN and PAN/O-MMT composite nanofibrous membranes coated with $\mathrm{TiO}_{2}$ films were prepared and used for $\mathrm{MB}$ adsorption and photodegradation. The effects of $\mathrm{O}$ MMT amount concentration and $\mathrm{TiO}_{2}$ spin coating layers on the structural morphology, adsorption, and photocatalytic properties of nanofiber membranes were investigated. The results revealed that with the increase of O-MMT concentration, the membranes showed better adsorption of $\mathrm{O}$ MMT and have no effect on the photocatalytic degradation. Besides, when the number of film layers increased, the adsorption process of $\mathrm{MB}$ was slowed down, but the photocatalytic ability was greatly improved. And this kind of materials have potential prospect in water treatment polluted by cationic dye.

\section{Acknowledgments}

This work was supported by the Specialized Research Fund for the Innovation Project of Jiangsu Graduate Education (no. CXZZ11_0471), the Doctoral Program of higher education (no. 20090093110004), the Natural Science Foundation of Jiangsu Province (no. BK2010140), and the scientific and technological guidance project of China National Textile and Apparel Council (no. 2011014).

\section{References}

[1] T. P. S. Sumanjit and W. K. Ravneet, "Removal of health hazards causing acidic dyes from aqueous solutions by the process of adsorption," Online Journal of Health and Allied Sciences, vol. 6, no. 3, article 3, 2007.

[2] A. Latif, S. Noor, Q. M. Sharif, and M. Najeebullah, "Different techniques recently used for the treatment of textile dyeing effluents: a review," Journal of the Chemical Society of Pakistan, vol. 32, no. 1, pp. 115-124, 2010.

[3] M. X. Zhu, L. Lee, H. H. Wang, and Z. Wang, "Removal of an anionic dye by adsorption/precipitation processes using alkaline white mud," Journal of Hazardous Materials, vol. 149, no. 3, pp. 735-741, 2007.

[4] P. Pengthamkeerati, T. Satapanajaru, N. Chatsatapattayakul, P. Chairattanamanokorn, and N. Sananwai, "Alkaline treatment of biomass fly ash for reactive dye removal from aqueous solution," Desalination, vol. 261, no. 1-2, pp. 34-40, 2010.

[5] P. Janoš, H. Buchtová, and M. Rýznarová, "Sorption of dyes from aqueous solutions onto fly ash," Water Research, vol. 37, no. 20, pp. 4938-4944, 2003.

[6] M. Özacar and I. A. Şengil, "Adsorption of reactive dyes on calcined alunite from aqueous solutions," Journal of Hazardous Materials, vol. 98, no. 1-3, pp. 211-224, 2003.

[7] S. Wang and Z. H. Zhu, "Sonochemical treatment of fly ash for dye removal from waste water," Journal of Hazardous Materials, vol. 126, pp. 91-95, 2005.
[8] K. Saeed, S. Haider, T. J. Oh, and S. Y. Park, "Preparation of amidoxime-modified polyacrylonitrile (PAN-oxime) nanofibers and their applications to metal ions adsorption," Journal of Membrane Science, vol. 322, no. 2, pp. 400-405, 2008.

[9] Z. Ma, K. Masaya, and S. Ramakrishna, "Immobilization of Cibacron blue F3GA on electrospun polysulphone ultra-fine fiber surfaces towards developing an affinity membrane for albumin adsorption," Journal of Membrane Science, vol. 282, no. 1-2, pp. 237-244, 2006.

[10] Z. Ma, M. Kotaki, and S. Ramakrishna, "Electrospun cellulose nanofiber as affinity membrane," Journal of Membrane Science, vol. 265, no. 1-2, pp. 115-123, 2005.

[11] R. Y. Lin, B. S. Chen, G. L. Chen, J. Y. Wu, H. C. Chiu, and S. Y. Suen, "Preparation of porous PMMA/Na+-montmorillonite cation-exchange membranes for cationic dye adsorption," Journal of Membrane Science, vol. 326, no. 1, pp. 117-129, 2009.

[12] A. H. Gemeay, A. S. El-Sherbiny, and A. B. Zaki, "Adsorption and kinetic studies of the intercalation of some organic compounds onto Na+-montmorillonite," Journal of Colloid and Interface Science, vol. 245, no. 1, pp. 116-125, 2002.

[13] C. C. Wang, L. C. Juang, T. C. Hsu, C. K. Lee, J. F. Lee, and F. C. Huang, "Adsorption of basic dyes onto montmorillonite," Journal of Colloid and Interface Science, vol. 273, no. 1, pp. 8086, 2004.

[14] H. Ichinose, M. Terasaki, and H. Katsuki, "Properties of peroxotitanium acid solution and peroxo-modified anatase sol derived from peroxotitanium hydrate," Journal of Sol-Gel Science and Technology, vol. 22, no. 1-2, pp. 33-40, 2001.

[15] W. Y. Gan, H. Zhao, and R. Amal, "Photoelectrocatalytic activity of mesoporous TiO2 thin film electrodes," Applied Catalysis A, vol. 354, no. 1-2, pp. 8-16, 2009.

[16] Z. Liuxue, L. Peng, and S. Zhixing, "Photocatalysis anatase thin film coated PAN fibers prepared at low temperature," Materials Chemistry and Physics, vol. 98, no. 1, pp. 111-115, 2006.

[17] B. Mahltig and T. Textor, "Combination of silica sol and dyes on textiles," Journal of Sol-Gel Science and Technology, vol. 39, no. 2, pp. 111-118, 2006.

[18] D. Juan, Z. Li, and C. Shuilin, "Wash fastness of dyed fabric treated by the sol-gel process," Coloration Technology, vol. 121, no. 1, pp. 29-36, 2005.

[19] H. Qiao, Y. Cai, F. Chen et al., "Influences of organic-modified Fe-montmorillonite on structure, morphology and properties of polyacrylonitrile nanocomposite fibers," Fibers and Polymers, vol. 10, no. 6, pp. 750-755, 2010.

[20] Y. Cai, Q. Li, Q. Wei, Y. Wu, L. Song, and Y. Hu, "Structures, thermal stability, and crystalline properties of polyamide6/organic-modified Fe-montmorillonite composite nanofibers by electrospinning," Journal of Materials Science, vol. 43, no. 18 , pp. 6132-6138, 2008.

[21] J. P. Zhang, L. Wang, and A. Q. Wang, "Preparation and properties of Chitosang-poly-(acrylic acid)/montmorillonite superabsorbent nanocomposite via in situ intercalative polymerization," Industrial and Engineering Chemistry Research, vol. 46, pp. 2497-2502, 2007.

[22] L. Wang, J. Zhang, and A. Wang, "Removal of methylene blue from aqueous solution using chitosan-g-poly(acrylic acid)/ montmorillonite superadsorbent nanocomposite," Colloids and Surfaces A, vol. 322, no. 1-3, pp. 47-53, 2008.

[23] G. Akçay and M. K. Yurdakoç, "Nonyl- and dodecylamines intercalated bentonite and illite from Turkey," Turkish Journal of Chemistry, vol. 23, no. 1, pp. 105-113, 1999. 


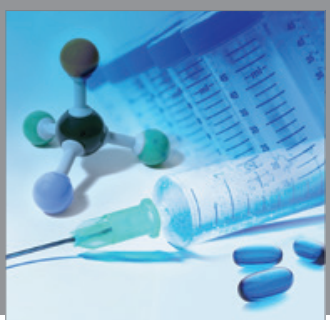

International Journal of

Medicinal Chemistry

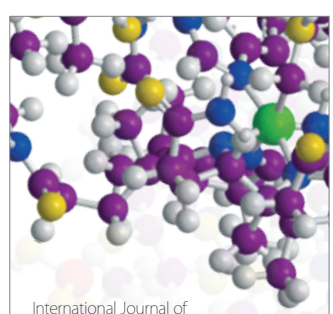

Carbohydrate Chemistry

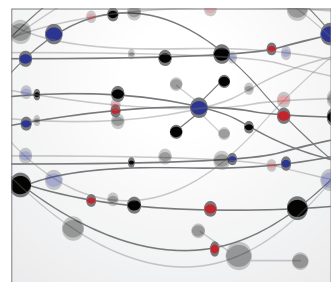

The Scientific World Journal
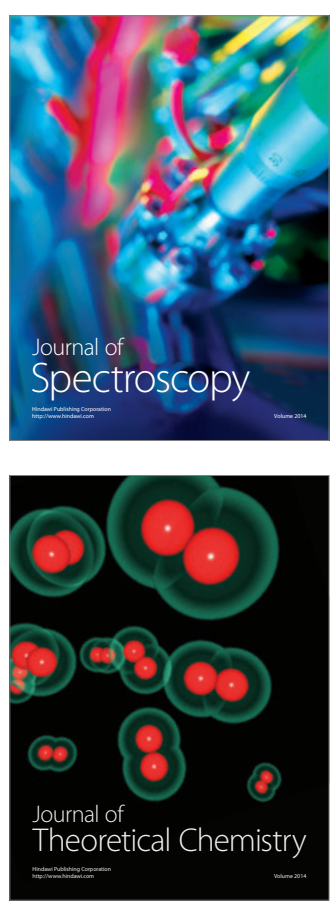
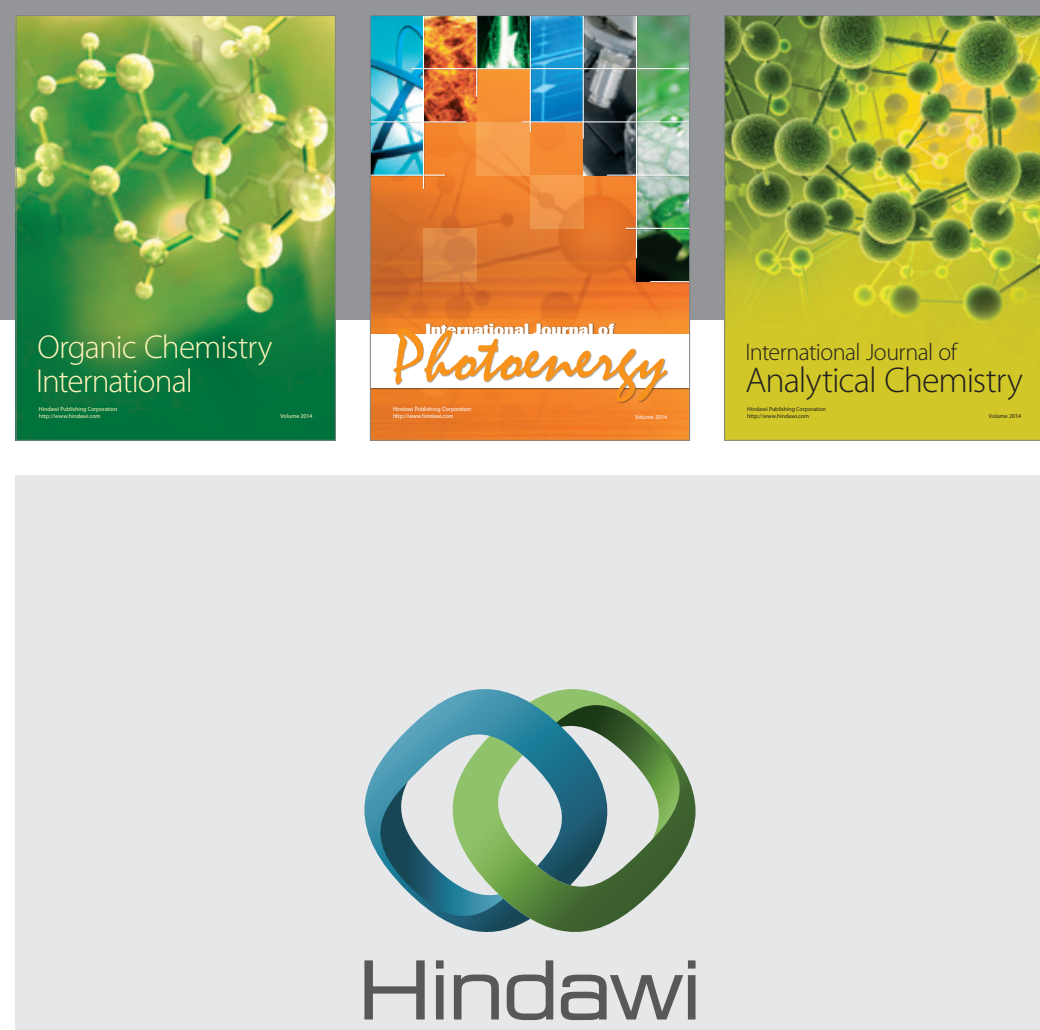

Submit your manuscripts at

http://www.hindawi.com
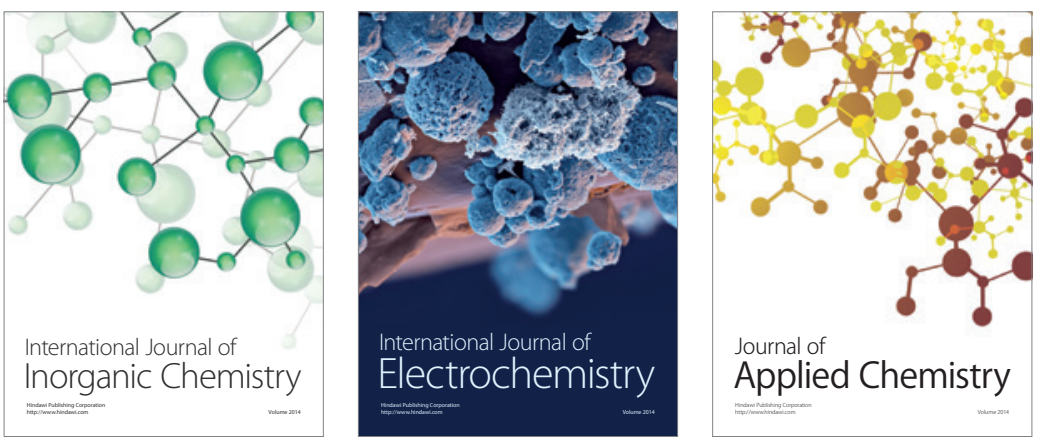

Journal of

Applied Chemistry
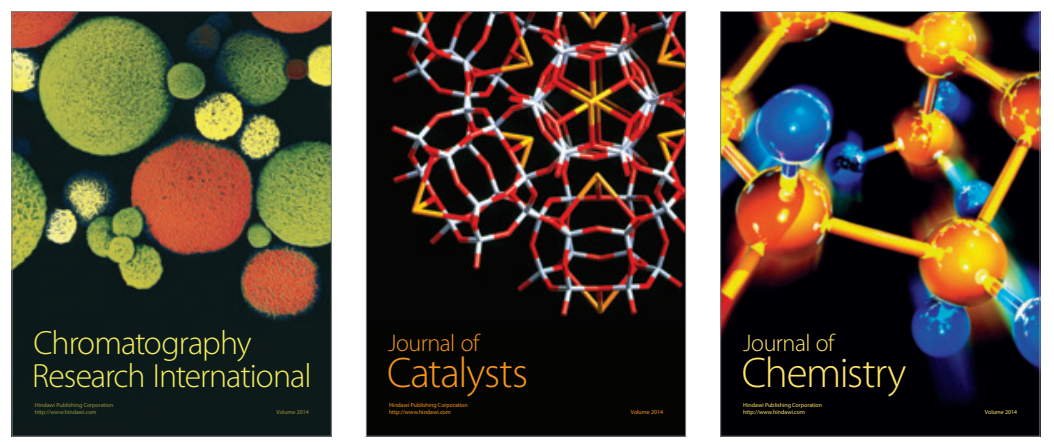
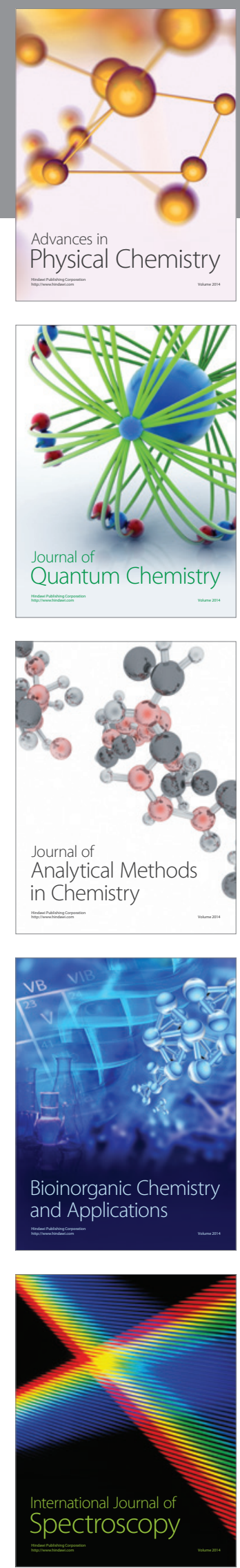